\title{
A Fast and Practical Contour Completion Approach with Chord- to-Point Distance
}

\author{
Yumin Dou, Mao Ye ${ }^{*}$, Renjie Huang, Xudong Li, Jiao Bao \\ School of Computer Science and Engineering, \\ Center for Robotics, \\ University of Electronic Science and Technology of China, \\ Chengdu, 611731, P.R. China \\ cvlabuestc@163.com
}

\begin{abstract}
As a kind of critical information in images and computer vision tasks, contour has a general application due to the robustness to illumination. However, it is far from meeting the current requirement because of expensive computation and noise in the real-world application. This paper proposes a novel approach of extracting contours in images. Firstly, we can obtain the salient points on the target contour by use of the accuracy property of Chord-to-point distance under combination of an ellipse model method. Then, a hyperbolic curve segment is used to fit the salient points, which can represent the target contour. The extensive experiments show that our method has better robustness and gives more exact approximation to the original target contour. Our work provides more selections for the practical application of contour completion.
\end{abstract}

Keywords: Salient point, ellipse model method, hyperbolic curve

\section{Introduction}

Contour can efficiently represent image structures with large spatial extents. Compared to other image cues, the shape contour is invariant to lighting conditions and variations in object color and texture. In recent years contour has attracted more and more attention in computer graphics, visualization and computer vision. There mainly exist two important stages in the process of extracting contour in an image: extracting the key points and fitting the curves based on the extracted points. On one hand, in the previous work, many researchers $[15,16]$ have studied how to represent contour with small amount of points through improving the accuracy of extracting points; on the other hand, the other researchers focus on the robustness of extracting the contour.

On the basis of many contour representations and description techniques developed in the past, a number of new techniques have been proposed in recent years. Chain code [15-17] describes an object by use of a sequence of unit-size line segments with a given orientation, which usually has high dimensions and is sensitive to noise. Hausdorff distance is a conventional method based on correspondence shape matching, and it has been often used to locate objects in an image and measure similarity between shapes boundary information. Shape matching approach with Hausdorff distance is also sensitive to slight variations and noise $[9,10]$. Except for high matching cost, shape signatures are sensitive to noise, and the large errors in matching may be caused by slight changes in the boundary.

Therefore, it is an undesirable method to make use of a shape signature to directly describe shape; in order to increase its robustness and reduce the matching load, a further processing is necessary. For example, a shape signature can be simplified by quantizing

${ }^{*}$ Corresponding Author 
the signature into a signature histogram, which is rotationally invariant. The scale space analysis is applied in consideration of problem of noise sensitivity and boundary variations in most spatial domain shape methods [7-11]. The scale space representation of a shape is created by tracking the position of inflection points in a shape boundary filtered by low-pass Gaussian filters of variable widths. However, the inflection point always deviates from the real position of the points due to the Gaussian filter $[12,13,18]$.

In order to extract the more exact points, Y. Zheng et. al, proposed an approach by computing topological persistence for a function defined on a Jordan curve in [14]. However, their method can't have a better performance due to a fixed threshold if the robustness to noise and clutter is considered. Compared to the topological persistence method in [14], the method of extracting salient points has a better performance under the same condition.

Moreover, because of various local variation and noise as well as different amounts of possibly smoothing parameters, only one variance parameter value may not be suitable for all curves of the same length. As a curvature estimation technique, the multi-scale detector based on chord-to-point distance accumulation (CPDA) can overcome these problems [4]. The primary advantage of the CPDA is that the application of any derivatives can be avoided; besides, it is less sensitive to local variation and noise to select the chord-length $\mathrm{L}$. The experiments indicate that it is more robust than the existing CSS-based detectors $[4,6,7]$. However, it is an expensive operation due to the fact that the CPDA detector [4] estimated the CPDA curvature value at each point of a given curve. It will be a suitable selection if we can find a method to decrease the number of candidate points. Consequently, we can see that the computational cost of the CPDA detector by this way can be reduced considerably.

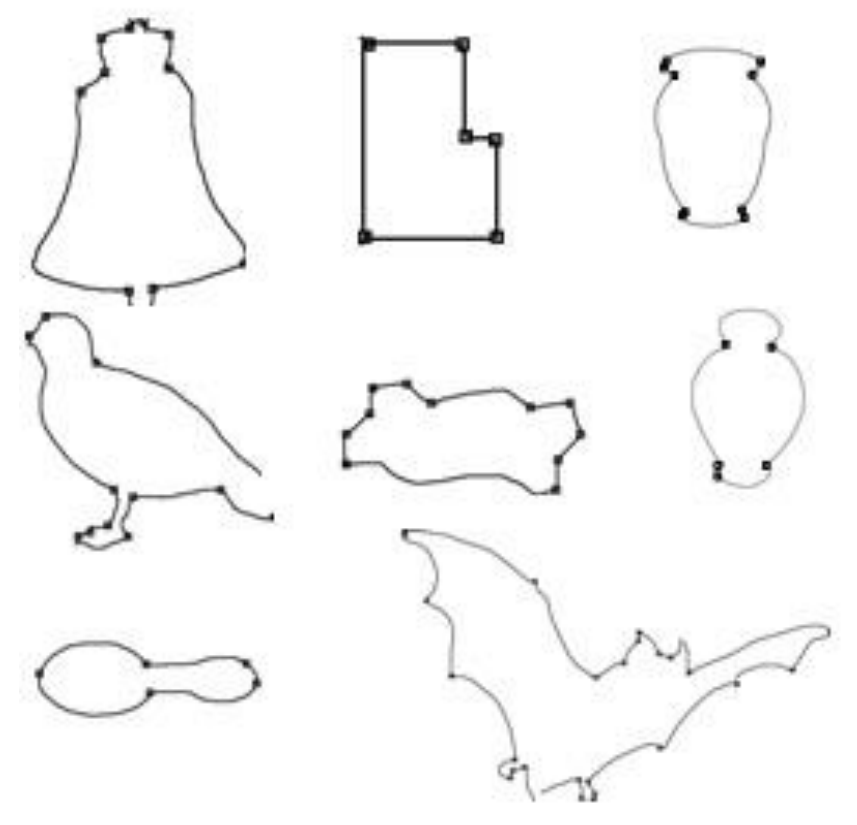

Figure 1. Corner Points Extracted by Fast CPDA

Our major contribution is the novel and practical contour completion approach in images which includes two stages. Firstly, we demonstrate the extraction salient point method by combining the CPDA technique and the model of ellipse planar curve through which an adaptive threshold is provided to improve the discriminative power in various situations. Secondly, we fit hyperbolic planar curves based on the obtained salient points by use of the least-squares method, so that the symmetry and asymmetry planar curve segments can be processed appropriately. 
This paper is organized as follows: Section 2 describes salient extraction approach; Section 3 presents an application of fitting curve method between adjacent salient points; the experimental results are discussed in Section 4; the final conclusion is given in Section 5.

\section{Salient Point Extraction}

Salient points consist of corner points and obtuse vertexes. It can be seen from Figure 1 , that the corner points only realize a poor representation of shape structure. An ellipse method shall be used to find more key points on the planar curve [5]. The process of extracting salient points is as illustrated in Figure 2. Firstly, an original curve is processed by use of distance function with two different parameters $\sigma 1, \sigma 2$ which represent Gaussian smoothing scale. Then the candidate points can be obtained and the chord-topoint distance is computed with each candidate point and it is able to obtain a set of corner points with filter. Finally, the ellipse planar curve model is applied to find the obtuse vertexes on the curve fragment between the adjacent corners.

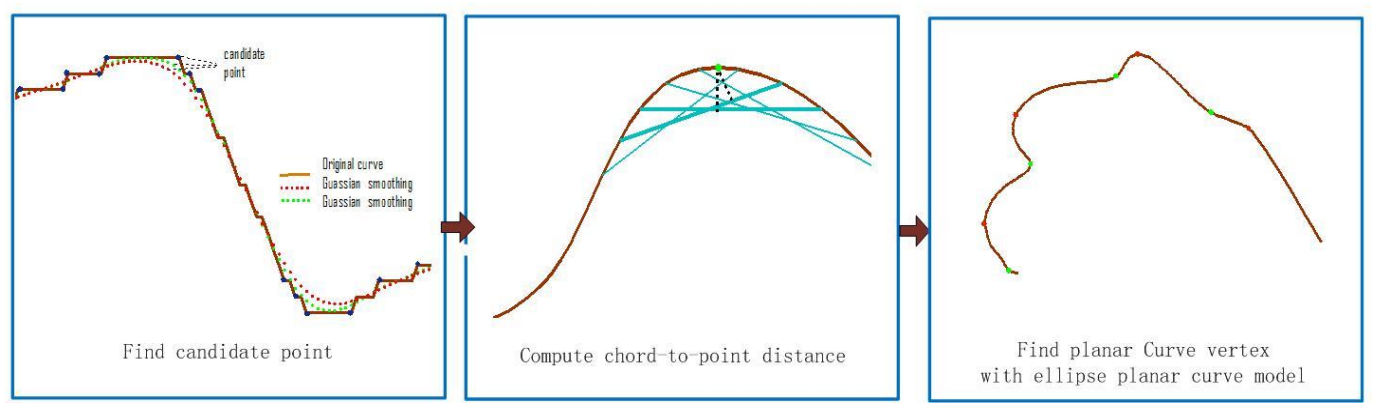

Figure 2. Algorithm Flow Chart of Extracting Salient Points

\subsection{Precise Detector by Use of Chord-to-Point Technique}

The CPDA detector works in four major steps, as discussed briefly in the following subsections. All chosen parameter values are decided based on existing work and empirical study $[1,5]$. Firstly, there are edge extraction and selection processes, that is to say, the Canny edge detector is used to extract planar curves from the grey image. Secondly, Gaussian convolution operations with different variances are conducted on the curves to smooth it. Thirdly, as the critical factor, the accumulation curvature estimation is used from a moving chord along a curve to each point on the curve. Lastly, it is required to refine the candidate corner set and remove the false corner.

However, the seeking process is complicated with high computational cost, for all points on the curve need to be processed. In order to reduce the spending time, it is considered to apply a method to simplify the process of each point. Through improvement of CPDA it is able to obtain a set of probable candidate points before CPDA curvature estimation. The basic step is to choose the critical points. Awrangjeb et al. find the difference of the distance between a point on an original curve and the corresponding point on the smoothed curve, when an original curve is smoothed by Gaussian [1]. It can be seen at left most in Figure 2, that the distance becomes higher and vice versa when the point is nearer the position of corner.

As a result, Awrangjeb et. al., viewed the maxima of the distance function as point-topoint distances between the original and smoothed curves, on which the points are the set of the candidate points [1]. The curve segments are actually smoothed by two Gaussian filters with different scales c, $\sigma 2$ (where $\sigma 1<\sigma 2$ ) in [1] due to the complicated local variations and noise. Then we can find the maxima as the candidate points, which are the differences of corresponding locations on the two smooth curve segments. In the formula 
$L(\theta)=(x(\theta), y(\theta)), L(\theta)$ is a digital boundary curve in the image, and $(x(\theta), y(\theta))$ are the points on the curve,

$L(\theta, \sigma) \otimes G(\theta, \sigma)=(x(\theta) \otimes G(\theta, \sigma), y(\theta) \otimes G(\theta, \sigma))$,

where $\otimes$ is defined as a convolution operation and Gaussian function is

$G(\theta, \sigma)=\frac{1}{\sqrt{2 \pi \sigma^{2}}} \exp ^{-\theta^{2} / 2 \sigma^{2}}$,

where $\sigma$ is used as the smoothing-scale; therefore, the distance function is

$F_{d}\left(t, \sigma_{1}, \sigma_{2}\right)=\left[x(t) \otimes G\left(t, \sigma_{2}\right)-x(t) \otimes G\left(t, \sigma_{1}\right)\right]^{2}$

$+\left[y(t) \otimes G\left(t, \sigma_{2}\right)-y(t) \otimes G\left(t, \sigma_{1}\right)\right]^{2}$.

\subsection{Ellipse Planar Curve Approximation}

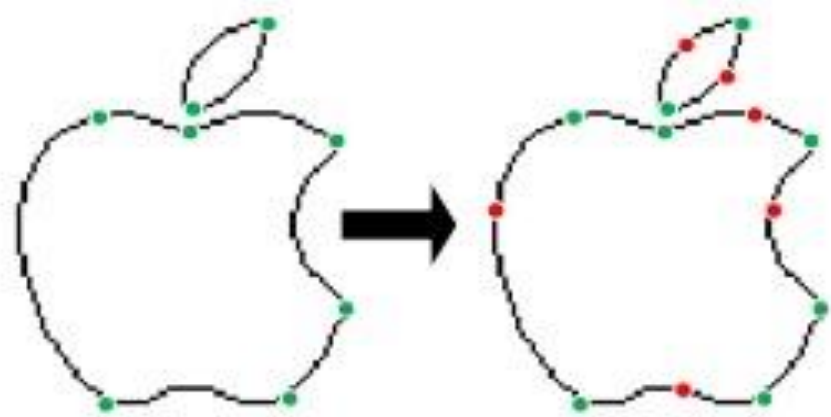

Figure 3. Left: Corner Points (Green) Extracted by Fast CPDA. Light: Obtuse Vertexes (red) by Ellipse Methods. Green and Red Points are Composed of Salient Points Proposed

It can be seen from the above, that all the works which have been done is to extract corner points. Generally, a well defined corner shall have a relatively sharp angle. In this paper, our task is to abstract the boundaries of image; however, the mere sharp corners are not enough to approximate the boundaries. In order to search for the obtuse vertex on the original curve between each adjacent corner point at the right most in Figure 2, an ellipse model approximation is applied [5]. An ellipse $\left.f(x)=\left[b^{2}-(b x / a)^{2}\right)^{(1 / 2)}\right]$ with $a$ and $b$ be the minor and major axis respectively is given. When the curvature $K$ of the vertex $(0, b)$ meets the threshold constraint, we regard the vertex as an obtuse vertex, which is needed to approximate the boundary better. The curvature function $K(x)$ is given as follow:

$$
\begin{aligned}
& K(x)=\frac{\left|f^{\prime \prime}(x)\right|}{1+\left|f^{\prime}(x)^{(3 / 2)}\right|} \\
& =\frac{b a^{4}}{\left[(b x)^{2}-(a x)^{2}+a^{4}\right]^{(3 / 2)}} .
\end{aligned}
$$

As a result,

$$
K_{\max }=K(0)=b / a^{2}, K_{\min }=K(a)=a / b^{2} .
$$


It is not enough to describe the boundary structure with the corners on the curve while there are various round arcs. In order to better approximate the contours, an ellipse approach is proposed to find the obtuse vertexes in Figure 3[5]. But different from [5], for finding local extreme points on curve fragments, we will find the vertex on curve fragments between two adjacent points. The global curvature characteristic of the neighbors is considered. In order to stably distinguish the obtuse vertex from the single point curvature, we find obtuse vertex in the region of support, which is to compute the area under the curve ranges from a value.

From [5], via the sum of point curvature range of curve segment, we can decide whether it is an obtuse vertex compared to an adaptive threshold. The integral is shown as follow,

$\int K(x) d x=\frac{b x}{\left[\left(b^{2}-a^{2}\right) x^{2}+a^{4}\right]^{(1 / 2)}}$.

From all the above, we can obtain corner points and obtuse vertexes, both of which are called salient points. As shown in Figure 4, the corner position of the smoothing curve deviates from the original position by use of CSS approach with different $\sigma$ of Gaussian, and CPDA can give a stable result at the position with various chord length $L \mathrm{~s}$. In order to find the vertex between adjacent corners [5], we can utilize a threshold $T$, with $T=R^{*} K$, which is a linear relationship with $K$. In this paper, we can see $K(x) \propto K_{L}$ approximately in Figure 4; therefore, we use the curvature $K_{L}(t)$ of CPDA rather than $K(x)$ of CSS due to its better robust property in our approach, thus the form $T=R^{*} K_{L}$ can be obtained. As can be seen in Figure 5, the shape contours are reconstructed accurately by using salient point method compared to [14].

\section{Fitting Curves between Adjacent Salient Points}

In this section, our task is to fit the planar curve segments between the adjacent salient points. After extraction of the contour segments, it can be seen that these planar curve segments are symmetrical or asymmetrical. However, most of them are asymmetrical.

Y. Zheng et. al., [14] used circle arc fragment to fit the segment which would give large fitting error due to it is symmetry. In order to alleviate this problem, we observe that the fitting error of the symmetrical or asymmetrical planar curve can be reduced by use of the hyperbola when approximating the planar curve segments.

As we know, the general form of hyperbola is given as follow:

$$
A x^{2}+B x y+C y^{2}+D x+E y+F=0 .
$$
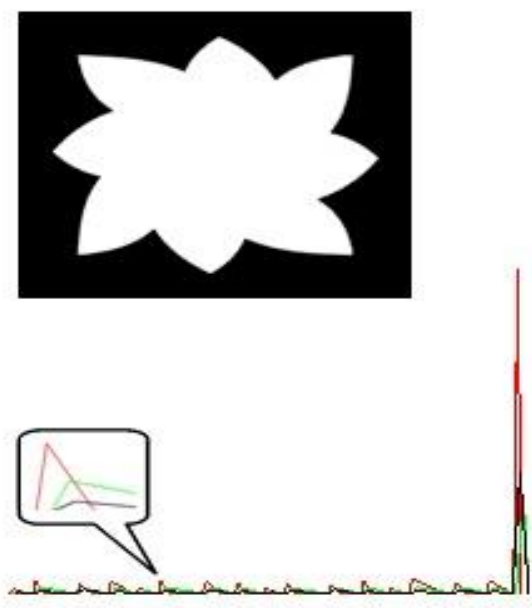


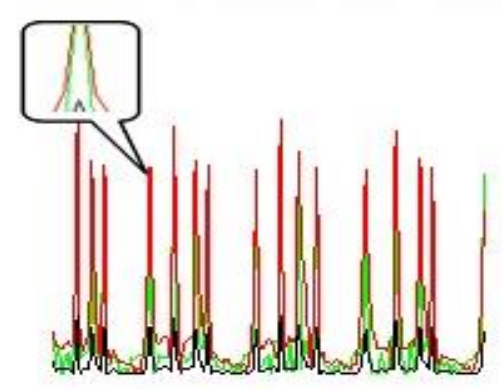

\section{Figure 4. Curvature with Multiple scales Processed. Top: Original Image.} Middle: Curvature with CSS. Bottom: Curvature with CPDA

In our case, we assume that $x, y$ are non-zero variables, thus the following formula can be obtained:

$$
A \frac{x}{y}+B+C \frac{y}{x}+\frac{D}{y}+\frac{E}{x}+\frac{F}{x y}=0
$$

Given that $A=C=F=0$, we have

$$
\frac{1}{y}=\frac{-B}{D}-\frac{E}{D} \frac{1}{x} \text {. }
$$

This is also a hyperbola. Given $\frac{1}{y}=u, \frac{1}{x}=v, t_{0}=\frac{-B}{D}, t_{1}=\frac{-E}{D}$ and $\mathbf{t}=\left(t_{0} t_{1}\right)^{T}$, the nonlinear problem is transformed into a linear problem.

Next, our task is to fit the planar curves by solving hyperbolic equation with the least square method [19]. Given the residuals $r_{j}=\phi\left(x_{j} ; t\right)-y_{j}(\mathrm{j}=1,2, \ldots, \mathrm{m})$, we can denote the residual vector as $\mathbf{r}=\left(\phi\left(x_{1}\right)-y_{1}, \phi\left(x_{2}\right)-y_{2}, \ldots, \phi\left(x_{m}\right)-y_{m}\right)^{T}$, and the sum of squares of each term in the residual vector $\mathbf{r}$ as follow:

$\mathrm{S}\left(t_{0}, t_{1}, \ldots, t_{n}\right)=\frac{1}{2} \sum_{i=1}^{m}\left[\sum_{j=0}^{n} t_{j} \phi_{j}\left(x_{i} ; \mathbf{t}\right)-y_{i}\right]^{2}$,

where $\mathrm{S}$ is a quadratic function of $t_{0}, t_{1}, \ldots, t_{n}$ as the independent variable. We need to find $t_{0}, t_{1}, \ldots, t_{n}$ to get the optimum of $\mathrm{S}$, which can make the residuals attain the minimum. When $\phi(x ; \mathbf{t})$ is a polynomial function, i.e., $\phi(x ; \mathbf{t})=t_{0}+t_{1} x+t_{2} x^{2}+$ $\ldots+t_{n} x^{n}=\sum_{j=0}^{n} t_{j} \phi_{j}(x ; \mathbf{t})$. An over-determined system can be obtained as follow:

$$
\left(\begin{array}{ccccc}
1 & \cdots & x_{1} & \cdots & x_{1}^{n} \\
\vdots & & \vdots & & \vdots \\
1 & \cdots & x_{k} & \cdots & x_{k}^{n} \\
\vdots & & \vdots & & \vdots \\
1 & \cdots & x_{m} & \cdots & x_{m}^{n}
\end{array}\right)\left(\begin{array}{c}
t_{0} \\
\vdots \\
t_{k} \\
\vdots \\
t_{n}
\end{array}\right)=\left(\begin{array}{c}
y_{1} \\
\vdots \\
y_{k} \\
\vdots \\
y_{m}
\end{array}\right)
$$


We take partial derivatives with respect to the independent variables $t_{0}, t_{1}, \ldots, t_{n}$ respectively and obtain as follow:

$$
\begin{aligned}
& \frac{\partial S}{\partial t_{0}}=\sum_{k=1}^{m}\left[\left(t_{0}+t_{1} x_{k}+\ldots+t_{n} x_{k}^{n}\right)-y_{k}\right] \\
& \frac{\partial S}{\partial t_{1}}=\sum_{k=1}^{m}\left[\left(t_{0}+t_{1} x_{k}+\ldots+t_{n} x_{k}^{n}\right)-y_{k}\right] x_{k} \\
& \vdots \\
& \frac{\partial S}{\partial t_{n}}=\sum_{k=1}^{m}\left[\left(t_{0}+t_{1} x_{k}+\ldots+t_{n} x_{k}^{n}\right)-y_{k}\right] x_{k}^{n} .
\end{aligned}
$$

It is given that partial derivatives to be zero. In order to fit the polynomial function $\phi(x)=t_{0}+t_{1} x+t_{2} x^{2}+\ldots+t_{n} x^{n}$, we need to solve a normal equation as follow:

$$
\left(\begin{array}{ccccc}
m & \cdots & \sum_{k=1}^{m} x_{k} & \cdots & \sum_{k=1}^{m} x_{k}^{n} \\
\vdots & & \vdots & & \vdots \\
\sum_{k=1}^{m} x_{k} & \cdots & \sum_{k=1}^{m} x_{k}^{2} & \cdots & \sum_{k=1}^{m} x_{k}^{n+1} \\
\vdots & & \vdots & & \vdots \\
\sum_{k=1}^{m} x_{k}^{n} & \cdots & \sum_{k=1}^{m} x_{k}^{\mathrm{n}+1} & \cdots & \sum_{k=1}^{m} x_{k}^{2 n}
\end{array}\right)\left(\begin{array}{c}
t_{0} \\
\vdots \\
\vdots \\
t_{k} \\
t_{n}
\end{array}\right)=\left(\begin{array}{c}
\sum_{k=1}^{m} y_{k} \\
\vdots \\
\sum_{k=1}^{m} x_{k} y_{k} \\
\vdots \\
\sum_{k=1}^{m} x_{k}^{n} y_{k}
\end{array}\right)
$$

In this case, the residuals $r_{j}(x)$ are linear, and the problem of minimizing is a linear least-squares problem. Therefore the residual vector can be written as $\mathbf{r}(x)=\mathbf{Q t}-\mathbf{U}$ for some matrix $\mathbf{Q}$ and vector $\mathbf{U}$, both of which are independent of $\mathrm{x}$, thus the objective function is

$$
S(\mathbf{t})=\frac{1}{2}\|\mathbf{Q} \mathbf{t}-\mathbf{U}\|^{2}
$$

When it is a linear fitting problem, recall the form (8) and let

$$
\mathbf{Q}=\left(\begin{array}{ccccc}
1 & \cdots & 1 & \cdots & 1 \\
x_{1} & \cdots & x_{k} & \cdots & x_{m}
\end{array}\right)^{T}, \mathbf{t}=\left(t_{0} t_{1}\right)^{T}, \mathbf{U}=\left(u_{1} u_{2}\right)^{T},
$$

As a result, we can obtain the normal equations which are transformed as a linear form as follow:

$\mathbf{Q}^{T} \mathbf{Q} \mathbf{t}=\mathbf{Q}^{T} \mathbf{U}$.

i.e., $\left(t_{0} t_{1}\right)^{T}=\left(\mathbf{Q}^{T} \mathbf{Q}\right)^{-1} \mathbf{Q}^{T} \mathbf{U}$.

According to the least square method, the coefficients $t_{0}$ and $t_{1}$ can be obtained as (9). It is able to obtain the form of fitting curves as follow through substituting $\mathbf{t}$ into (6):

$$
y(x)=\frac{x}{t_{0} x+t_{1}} .
$$


Next, in order to get the appropriate fitting value, the direction of the planar curve segments shall be determined. Firstly, we connect the two endpoints of the curve with a chord. Then we draw a line through the middle point of the chord, which is perpendicular to the chord and intersects at point $p$ with the curve. The curve is split into two parts at $p$, and we can obtain a sum of distances from points in each part to the line segment. Finally, compared with the two sums of distances, we can get the position of the peaks, which can be used to determine the start point of the hyperbola.
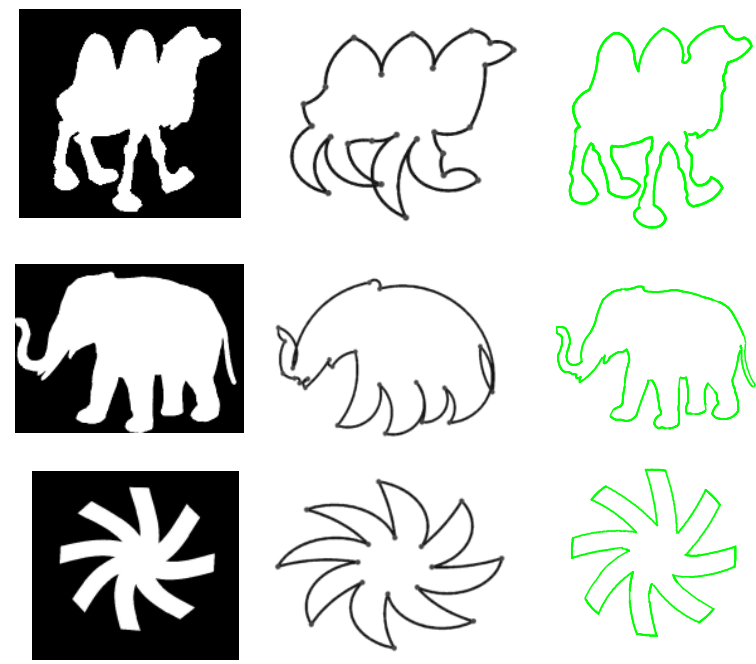

Figure 5. Left: Original Image; Middle: the Method from [14]; Right: Shape based on Salient Point Method

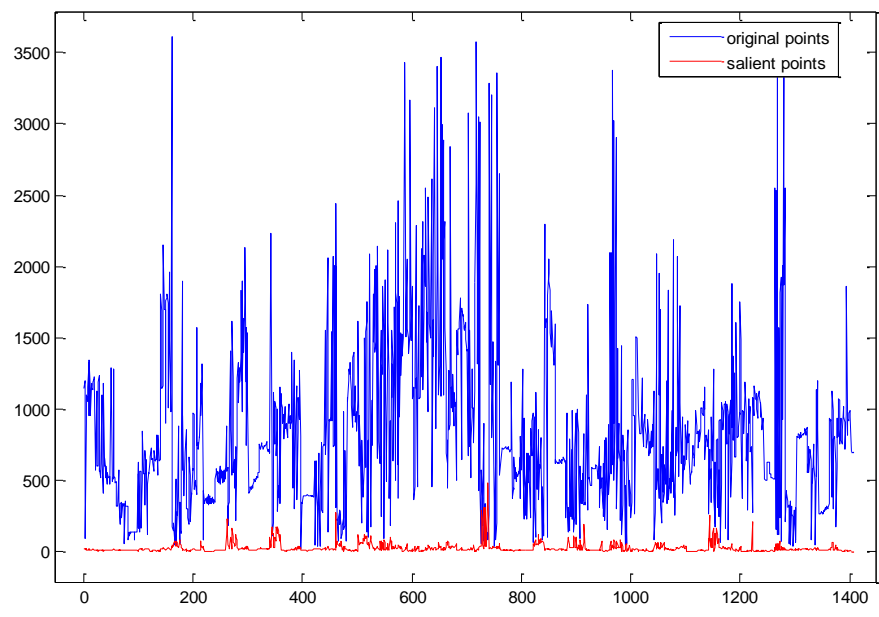

Figure 6. The number of original points in edge image of MPEG7 is compared to the number of salient points. Horizontal axis denotes each image index in MPEG7 dataset, vertical is the point number of the image.

\section{Experiments}

In this section, we conduct on two data sets based on our proposed approach, which are MPEG7 and ETHZ. MPEG7 is a silhouette database which composes of 70 classes, 
and each class has 20 different shapes, with 1400 contour examples in total. There are large variations with intra-class and similar inter-class for the various objects in the dataset.

The salient method is implemented as follows. Firstly, the canny operator and edgelinking algorithm are used to process the edge map, for purpose of obtaining the curves in edge map. Secondly, CPDA technique is applied to find the corners. According to the Gaussian method, we can find the candidate points of corner, then compute the distance of a moving chord to each point, and then we can get the vertex of planar curve via integrating the points between adjacent corners point and ellipse model. Finally, the fitting method of hyperbola is applied on both classes of points (as shown in Figure 8). It can be seen in Figure 6, that the number of salient points is reduced to less than 3 percents compared to the original curve point sequence, which largely simplifies the number of points in edge images.

In this section, we set the chord length $L$ as 10, 20, 30 respectively, and set the $R=2.6$, i.e., the auto threshold value for the vertexes between the adjacent corners. It can be observed from [14], that there are sharp extremities of the shape and the fitting outline has obvious distortion like arc fragment in Figure 5. However, there is a better performance as for curves approximation via partial hyperbola.

Another experiment is implemented on ETHZ dataset which contains five different classes, that is, apples, mugs, bottles, giraffes and swans. There are one or more objects in each image with 255 images in total. Meanwhile, the objects have large variations in viewpoint, size, appearance and non-rigid deformation. Since the nature scene images contains largely complicated boundaries in Figure 7, the image can be simplified with very small amount of points with the salient approach proposed which can significantly reduce the number of points in data storage or in transferring in communication.
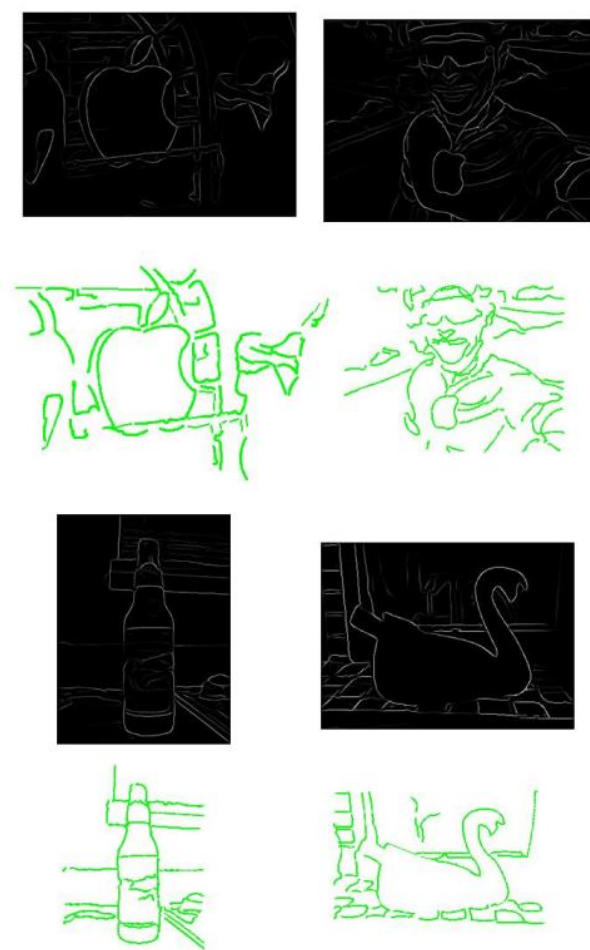
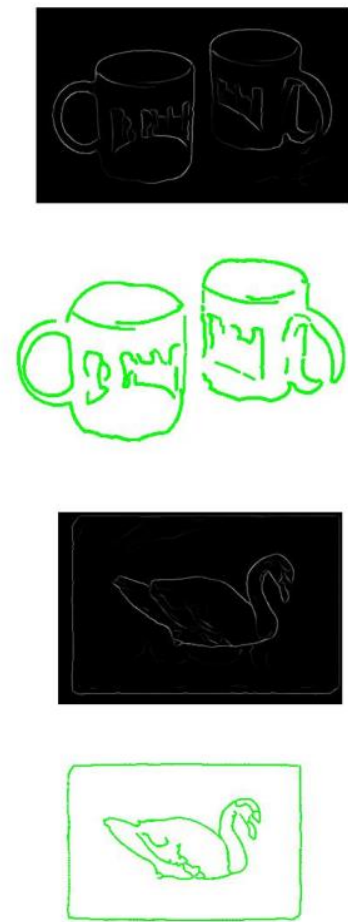
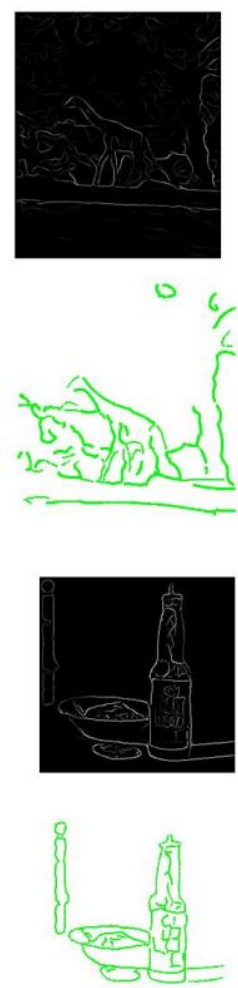

Figure 7. Contour Completion with Salient Point Method on ETHZ

These images in this dataset are natural scene. As a contour completion task, the sketching according to the image is complicated especially in natural scene. Topological 
persistence method [14] has a good performance in simple scene image, but the desired result can't be achieved in natural image. In addition, the running time cost has been largely increased. Only extreme points can be found in their method which sets a fixed threshold with only considering the local minimum property. It is required to depend on the curvature of more than one single point when the round corners are sought.
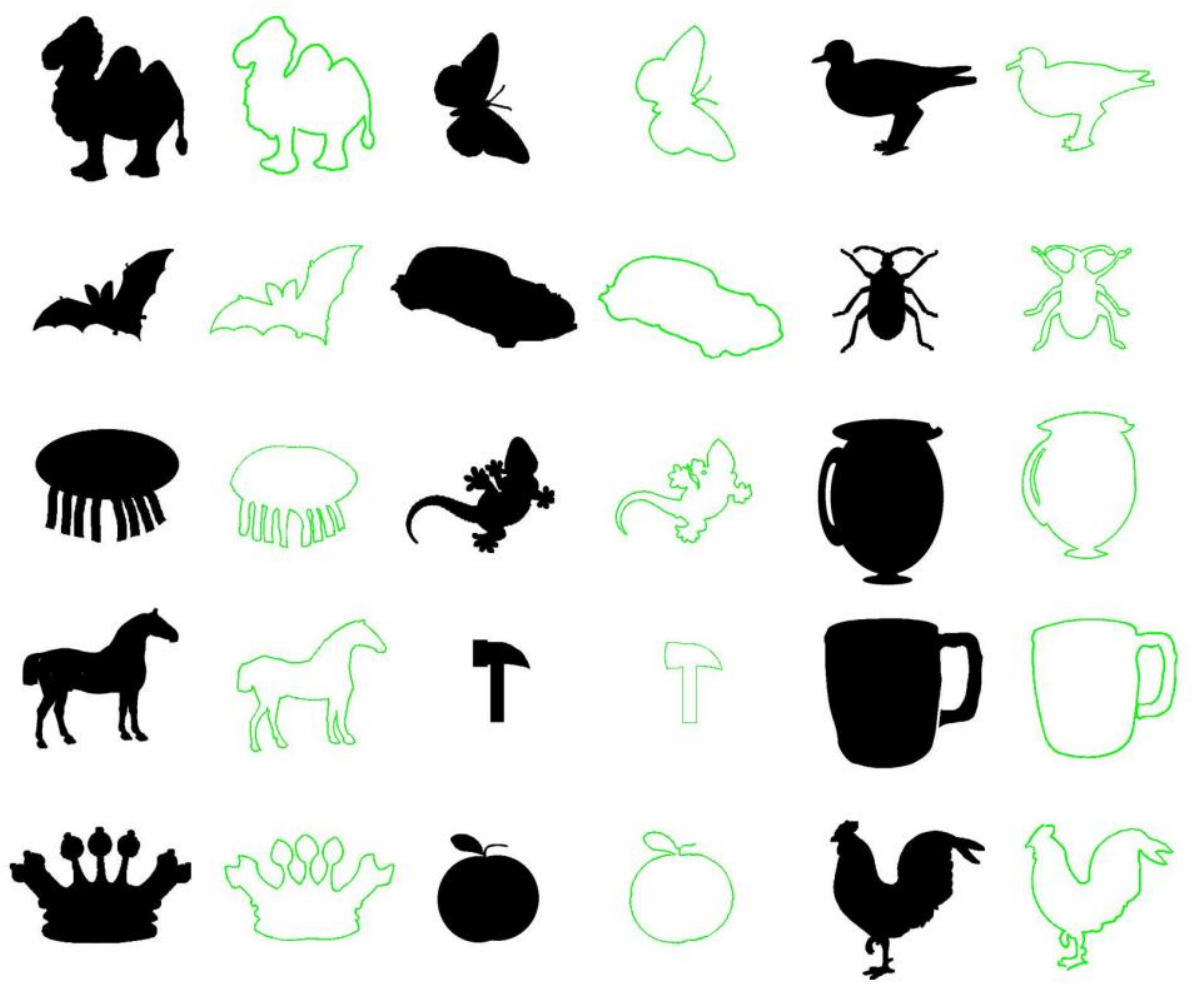

Figure 8. Shape Contour Abstraction based on Salient Points

There will be a failure in finding them. Despite of same curvature, they may be selected or not in different positions according to the situation. However, the fixed thresholds cannot meet the requirement because the points will be all selected or not. In consideration of the global properties of curves, the salient points method proposed are robustness to local trivial noise invariance.

\subsection{Computational Complexity}

In this section, the detailed discussion is given on the computation cost of our proposed method. In [1], Mohammad Awrangjeb et. al., described the total number of operations is $2 m n+n+408 n_{p}$, where $n$ is the number of obtained candidate points $\left(n_{p}<<n\right)$. Firstly, in order to detect the salient points, we add the seeking rounder corner method, but this method only increasing $n_{p}$ computing operation with relation to candidate points. Therefore, for the sake of the subcomponent of extracting salient points, it is $2 m n+n+409 n_{p}$. Secondly, the least square method is used in order for the fitting algorithm but only $n_{p}$ operation is increased, where $n_{p}$ is points on the boundary curve. As a result, the total compute complexity is $2 m n+n+409 n_{p}$.

The proposed algorithm was executed on a $2.20 \mathrm{GHz}$ pc with $2 \mathrm{G}$ memory. The whole procedure includes two stages that are extracting salient points and fitting curve segments. The first step is divided into two parts, i.e. seeking the sharp points and curves vertexes. The time of seeking salient points on the planar curves is as shown in Figure 7, 
excluding edge detection and contour extraction, the time varies from 0.0045 to $0.0086 \mathrm{~s}$ according to the size of image and the complexity of the image scene.

\section{Conclusion}

In this paper, we have proposed a novel approach of abstracting contour by combining salient point extraction and ellipse model. Moreover, the experimental results have confirmed that our approach is very effective and practical. In our work, we have creatively introduced the CPDA technique to salient point extraction, which can reduce the computation cost largely. Furthermore, we used the ellipse model instead of Jordan curve in previous methods in the process of representing the curves of target. In addition, we give consideration to global characteristic for the purpose of realizing more robust representation of target contour. In conclusions, our method makes contour abstraction more usable in many computer vision applications. In the future work, we will apply the approach to object outline contour detection and target boundary location.

\section{Acknowledgments}

This work was supported in part by the National Natural Science Foundation of China (61375038).

\section{References}

[1] M. Awrangjeb, G. Lu, C.S. Fraser and M. Ravanbakhsh, "A Fast Corner Detector Based on the Chordto-Point Distance Accumulation Technique", IEEE Conf. Digital Image Computing: Techniques and Applications ,(2009); Australia.

[2] D. DeMenthon, V. Kobla, and D. Doermann, "Video summarization by curve simplication", ACM Multi-media (1998).

[3] H. Etou, Y. Okada, and K. Niijima, "Feature preserving motion compression based on hierarchical curve simplification", IEEE International Conference on Multimedia and Expo, (2004);Taipei.

[4] J.H.Han, T. Poston.Chord-to-point distance accumulation and planar curvature: a new approach to discrete curvature.Pattern Recognition Letters, vol. 22(2001),pp. 1133-1144, 2001.

[5] X.C.He, N.H.C.Yung. Curvature Scale Space Corner Detector with Adaptive Threshold and Dynamic Region of Support. Proceedings of the 17th International Conference on Pattern Recognition,( 2004) Aug 23-26;Cambridge,UK

[6] V. Ferrari, T. Tuytelaars, and L. Van Gool. Object Detection with Contour Segment Networks. $9^{\text {th }}$ European Conference on Computer Vision,(2006)May 7-13;Graz,Austria

[7] F. Mokhtarian and R. Suomela. Robust image corner detection through curvature scale space. IEEE Trans. Pattern Anal. Mach. Intell.vol. 20, no. 12, pp. 1376C1381, Dec. (1998)

[8] F. Mokhtarian and F. Mohanna. Performance evaluation of corner detectors using consistency and accuracy measures. Computer Vision and Image Understanding, vol. 102, no. 1, pp. 81C94, Apr. (2006)

[9] F. Mokhtarian and A. K. Mackworth. Scale-based description and recognition of planar curves and two-dimensional shapes. EEE Trans. Pattern Anal. Mach. Intell, vol. 8, no. 1, pp. 34C43, (1986).

[10] X. Mi, D. DeCarlo, and M. Stone. Abstraction of 2d shapes in terms of parts. in NPAR,(2009)Aug 1-2, New Orleans, Louisiana, USA

[11] B. K. Ray and R. Pandyan. ACORD-an adaptive corner detector for planar curves. Pattern Recognistion, vol. 36, pp. 703C708,(2003)

[12] P. Saint-Marc, J. S. Chen, and G. Medioni. Adaptive smoothing: a general tool for early vision. IEEE Trans. on Pattern Analysis and Machine Intelligence, vol.13, no. 6, pp. 514C529, (1991)

[13] X. Zhang, M. Lei, D. Yang, Y. Wang, and L. Ma. Multi-scale curvature product for robust image corner detection in curvature scale space. Pattern Recognistion Letters, vol. 28pp. 545C554, (2007)

[14] Y. Zheng, S. Gu, and C. Tomasi. Topological persistence on a Jordan curve. In Proc. ICASSP, (2012)March 25-30; Kyoto,Japan

[15] Dengsheng. Zhang, and Guojun. Lu. Review of shape representation and description techniques. Pattern Recognition, vol. 37, pp. 1-19, (2004)

[16] H. Freeman. On the encoding of arbitrary geometric configurations.IRE Trans. Electron. Comput. EC10 (1961) $260 C 268$.

[17] P. Xu, M. Ye, X. Li, L. Pei and P. Jiao, "Object Detection Using Voting Spaces Trained by Few Samples", Optical Engineering, vol. 52, no. 9, (2013).

[18] R. Huang, T. Li, M. Ye and Y. Dou, "Unconstrained Face Verification by Optimally Organizing Multiple Classifiers", International Journal of Control, Automation and Systems, vol 12, no 4, (2014).

[19] J. Nocedal, S. J. Wright, "Numerical Optimization Second Edition”, Springer New York, (2006). 

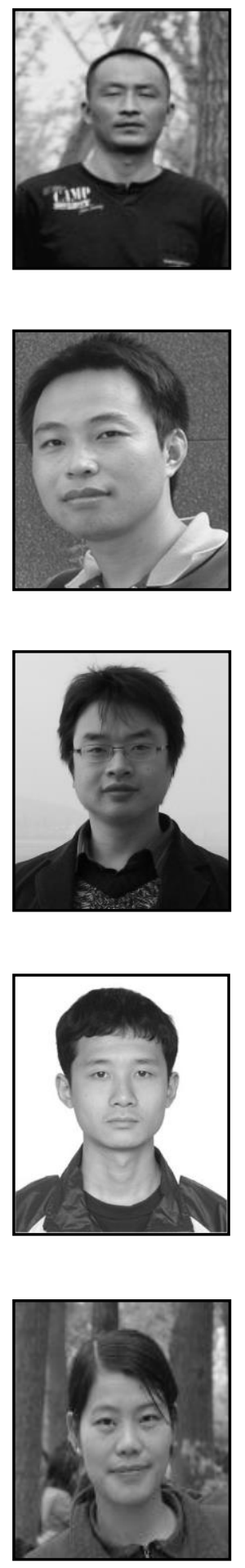

\section{Authors}

Yumin Dou, He received his master's degree in Computer Science from Chengdu University of Technology in 2010. He is currently a Ph.D. candidate at the School of Computer Science and Engineering of University of Electronic Science and Technology University of China. His research interests include pattern recognition and computer vision.

Mao Ye, He received his Ph.D. degree in Mathematics from Chinese University of Hong Kong, in 2002. He is currently a professor and Director of CVLab at University of Electronic Science and Technology of China. His current research interests include machine learning and computer vision. In these areas, he has published over 70 papers in leading international journals or conference proceedings.

Renjie Huang, He received his master's degree in Computer Science from Southwest University in China in 2006. He is currently a Ph.D. candidate at the School of Computer Engineering and Technology of University of Electronic Science and Technology of China. He is also an instructor at the School of Computer and Information Science of Southwest University in China. His research interests include machine learning and computer vision.

Xudong Li, He received his BS degree in mathematics from Chengdu University of Technology, Chengdu, China, in 2011. He has been taking the successive master-doctor program since September 2011. He is currently a $\mathrm{PhD}$ student in University of Electronic Science and Technology of China, Chengdu, China. His current research interests include machine learning and computer vision.

Jiao Bao, She received her master's degree in University of Electronic Science and Technology of China in 2011. She is currently a Ph.D. candidate at the School of Computer Engineering and Technology of University of Electronic Science and Technology of China. Her research interests include machine learning and computer vision. 